\title{
Racial differences in B cell receptor signaling pathway activation
}

\author{
Diane M Longo ${ }^{1 *}$, Brent Louie ${ }^{1}$, Kavita Mathi ${ }^{2}$, Zoltan Pos ${ }^{3}$, Ena Wang ${ }^{4}$, Rachael E Hawtin ${ }^{1}$, \\ Francesco M Marincola ${ }^{4}$ and Alessandra Cesano ${ }^{1}$
}

\begin{abstract}
Background: Single-cell network profiling (SCNP) is a multi-parametric flow cytometry-based approach that simultaneously measures basal and modulated intracellular signaling activity in multiple cell subpopulations. Previously, SCNP analysis of a broad panel of immune signaling pathways in cell subsets within PBMCs from 60 healthy donors identified a race-associated difference in B cell anti-IgD-induced PI3K pathway activity.

Methods: The present study extended this analysis to a broader range of signaling pathway components downstream of the $B$ cell receptor (BCR) in European Americans and African Americans using a subset of donors from the previously analyzed cohort of 60 healthy donors. Seven BCR signaling nodes (a node is defined as a paired modulator and intracellular readout) were measured at multiple time points by SCNP in PBMCs from 10 healthy donors [5 African Americans (36-51 yrs), 5 European Americans (36-56 yrs), all males].

Results: Analysis of BCR signaling activity in European American and African American PBMC samples revealed that, compared to the European American donors, B cells from African Americans had lower anti-lgD induced phosphorylation of multiple BCR pathway components, including the membrane proximal proteins Syk and SFK as well as proteins in the PI3K pathway (S6 and Akt), the MAPK pathways (Erk and p38), and the NF-KB pathway (NF$\mathrm{KB})$. In addition to differences in the magnitude of anti-lgD-induced pathway activation, racial differences in $\mathrm{BCR}$ signaling kinetic profiles were observed. Further, the frequency of IgD+ B cells differed by race and strongly correlated with BCR pathway activation. Thus, the race-related difference in BCR pathway activation appears to be attributable at least in part to a race-associated difference in IgD+ B cell frequencies.
\end{abstract}

Conclusions: SCNP analysis enabled the identification of statistically significant race-associated differences in BCR pathway activation within PBMC samples from healthy donors. Understanding race-associated contrasts in immune cell signaling responses may be one critical component for elucidation of differences in immune-mediated disease prevalence and treatment responses.

Keywords: Multi-parameter flow cytometry, BCR signaling, Race

\section{Background}

Racial differences have been documented in the prevalence of autoimmune diseases such as systemic lupus erythematosus [1] and multiple sclerosis [2] and in the clinical response to immunotherapies [such as IFN- $\alpha$ [3] and Benlysta/belimumab [4]]. However, the biologic basis for such race-associated differences remains poorly understood. A better understanding of the

\footnotetext{
* Correspondence: diane.longo@nodality.com

'Nodality, South San Francisco, CA 94080, USA

Full list of author information is available at the end of the article
}

underlying biologic mechanisms of race-associated differences in immune signaling responses may provide clinically relevant information regarding the mechanisms underlying race-related differences in treatment responsiveness.

Single-cell network profiling (SCNP) is a multiparametric flow cytometry-based approach that enables the simultaneous measurement of basal and evoked signaling in multiple cell subpopulations [5]. Recently, SCNP technology was applied to quantify immune signaling pathway activation following modulation with 12 immunomodulators (including IFN- $\alpha$, IFN- $\gamma$, IL-2, IL-4,

\section{Biomed Central}


Table 1 Summary of donor numbers, age, race, and gender

\begin{tabular}{lcc}
\hline & AA & EA \\
\hline No. of Donors & 5 & 5 \\
Mean Age (y) & 45.4 & 48.6 \\
Age Range (y) & $36-51$ & $36-56$ \\
Gender & 5 Male & 5 Male \\
\hline
\end{tabular}

IL-6, IL-10, IL-27, anti-IgD, LPS, R848, PMA, and CD40L) in 7 distinct immune cell subpopulations within PBMC samples from 60 healthy donors [6]. Using a training/test set approach, race-associated differences in anti-IgD-induced levels of $\mathrm{p}-\mathrm{S} 6$ and $\mathrm{p}$-Akt in B cells were identified [6]. The present study was performed to analyze anti-IgD-induced modulation of a broader range of BCR signaling pathway components at multiple time points using a subset of European American (EA) and African American (AA) donor samples from the previously analyzed healthy donor cohort [7].

\section{Methods}

\section{PBMC samples}

Cryopreserved PBMC samples collected from 10 healthy donors [5 AAs (mean age 45.4 yrs), 5 EAs (mean age 48.6 yrs), all males (Table 1)] within the Department of Transfusion Medicine, Clinical Center, National Institutes of Health with Institutional Review Board approval were used in this study. All blood samples, donated for research purposes with informed consent, were collected and processed as described previously [8].

\section{SCNP assay}

Cryopreserved PBMC samples were thawed at $37^{\circ} \mathrm{C}$ and resuspended in RPMI 1640 (1\% FBS) before staining with amine aqua viability dye (Invitrogen, Carlsbad, CA). Cells were resuspended in RPMI 1640 (10\% FBS), aliquoted to
100,000 cells per well in 96-well plates, and rested for $2 \mathrm{~h}$ at $37^{\circ} \mathrm{C}$ prior to incubation with anti-IgD $5 \mu \mathrm{g} / \mathrm{ml}$ (BD, San Jose, CA) or anti-IgM $10 \mu \mathrm{g} / \mathrm{ml}$ (Southern Biotech, Birmingham, AL). After modulation with anti-IgD (for $5^{\prime}, 15^{\prime}, 30^{\prime}$, or $60^{\prime}$ ) or anti-IgM $\left(5^{\prime}\right)$, cells were fixed with paraformaldehyde and permeabilized with 100\% ice-cold methanol as previously described [9]. Permeabilized cells were washed with FACS buffer (PBS, 0.5\% BSA, $0.05 \% \mathrm{NaN}_{3}$ ), pelleted, and stained with fluorochrome-conjugated Abs. Abs used include anti-CD20 (clone H1), -p-NF-kB (clone K10-895.12.50), -c-poly (ADP-ribose) polymerase (clone F21-852), -p-Erk (clone 20A), -p-SFK/Lck (clone 4/LckY505), -p-p38 (clone 36/ p38), -p-Syk (clone 17a/P-ZAP70) [BD, San Jose CA]; -pAkt (clone D9E), -p-S6 (clone 2F9) [CST, Danvers, MA]; and -IgD [Southern Biotech, Birmingham, AL].

\section{Flow cytometry data acquisition and analysis}

Flow cytometry data was acquired using FACS DIVA software (BD, San Jose, CA) on an LSRII Flow Cytometer (BD, San Jose, CA). All flow cytometry data were analyzed with WinList (Verity House Software, Topsham, $\mathrm{ME})$. For all analyses, dead cells and debris were excluded by forward scatter (FSC), side scatter (SSC), and amine aqua viability dye. Viable B cells were delineated according to the gating scheme shown in Figure 1.

\section{SCNP terminology and metrics}

The term "signaling node" refers to a specific protein readout in the presence or absence of a specific modulator. For example, the response to anti-IgD stimulation can be measured using $\mathrm{p}-\mathrm{S} 6$ as a readout. This signaling node is designated "anti-IgD $\rightarrow \mathrm{p}-\mathrm{S} 6$ ". The "Fold" metric was applied to measure the level of anti-IgD-induced modulation of each signaling molecule after modulation compared to its level in the basal state. The "Fold" metric

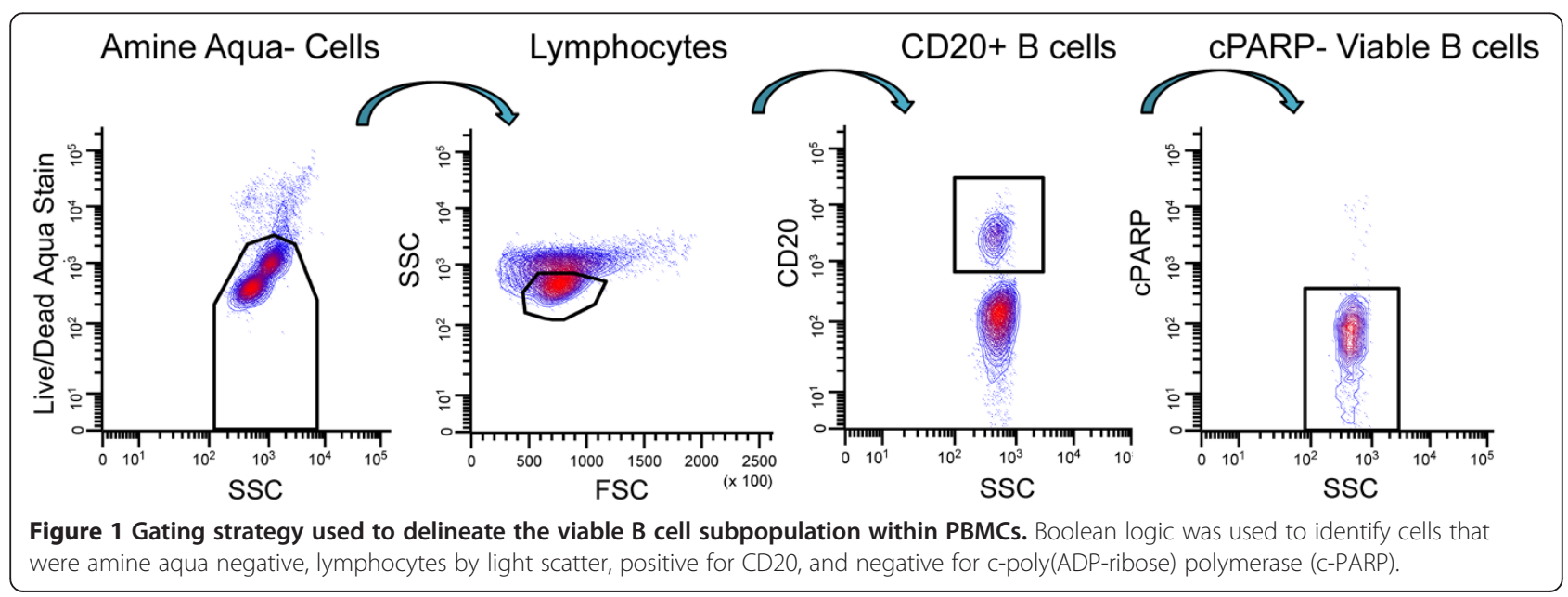


was calculated as follows:

Fold : $\left(\log _{2}[\operatorname{MFI}(\right.$ Modulated $) / \operatorname{MFI}($ Unmodulated $\left.)]\right)$

Where MFI is the median fluorescence intensity.

\section{Statistical analyses}

Statistical differences between groups were assessed by the Wilcoxon rank sum test. For each of the 7 phosphoprotein readouts, race-associated differences were assessed by averaging the Fold values over the 4 modulated time points $(5,15,30,60$ ) and applying the Wilcoxon rank sum test to the averaged Fold value. One donor was excluded from all analyses due to an insufficient number $(<200)$ of B cells ("events") collected by the flow cytometer.

\section{Correlations between signaling nodes}

$\mathrm{R}$ software (version 2.12.1) was used to compute Pearson correlation coefficients between IgD $+B$ cell frequency and BCR signaling nodes. Heatmaps were generated in Excel 2007 (Microsoft, Redmond, WA).

\section{Results and discussion}

Seven signaling nodes (anti-IgD $\rightarrow$ p-S6, anti-IgD $\rightarrow$ pSyk, anti-IgD $\rightarrow$ p-Akt, anti-IgD $\rightarrow$ p-Erk, anti-IgD $\rightarrow$ $\mathrm{SFK}, \quad$ anti-IgD $\rightarrow \mathrm{p}-\mathrm{p} 38$, and anti-IgD $\rightarrow \mathrm{p}-\mathrm{NF}-\mathrm{kB}$ ), or specific protein readouts of modulated signaling, were measured in viable B cells (defined as amine aqua negative, forward scatter/side scatter low, CD20+, c-poly (ADP-ribose) polymerase (c-PARP) negative cells, Figure 1) at several time points following modulation with anti-IgD in PBMCs collected from 10 healthy donors (Table 1). The "Fold" metric (see Materials and Methods) was applied to measure the level of a signaling molecule after modulation compared to its level in the basal state.

Averaged over all donors, viable $\mathrm{B}$ cells constituted $18.3 \%$ (standard deviation $=8.8 \%$, range $=2.2-28.1 \%$ ) of the parental Lymphocyte subpopulation (see Figure 1). For one donor, the CD20+ B cell percentage was extremely low (2.2\% of Lymphocytes) resulting in too few B cells for analysis (i.e. $<200 \mathrm{~B}$ cells in most wells of the 96-well plate). Therefore, data for this donor was excluded from the analysis presented below.

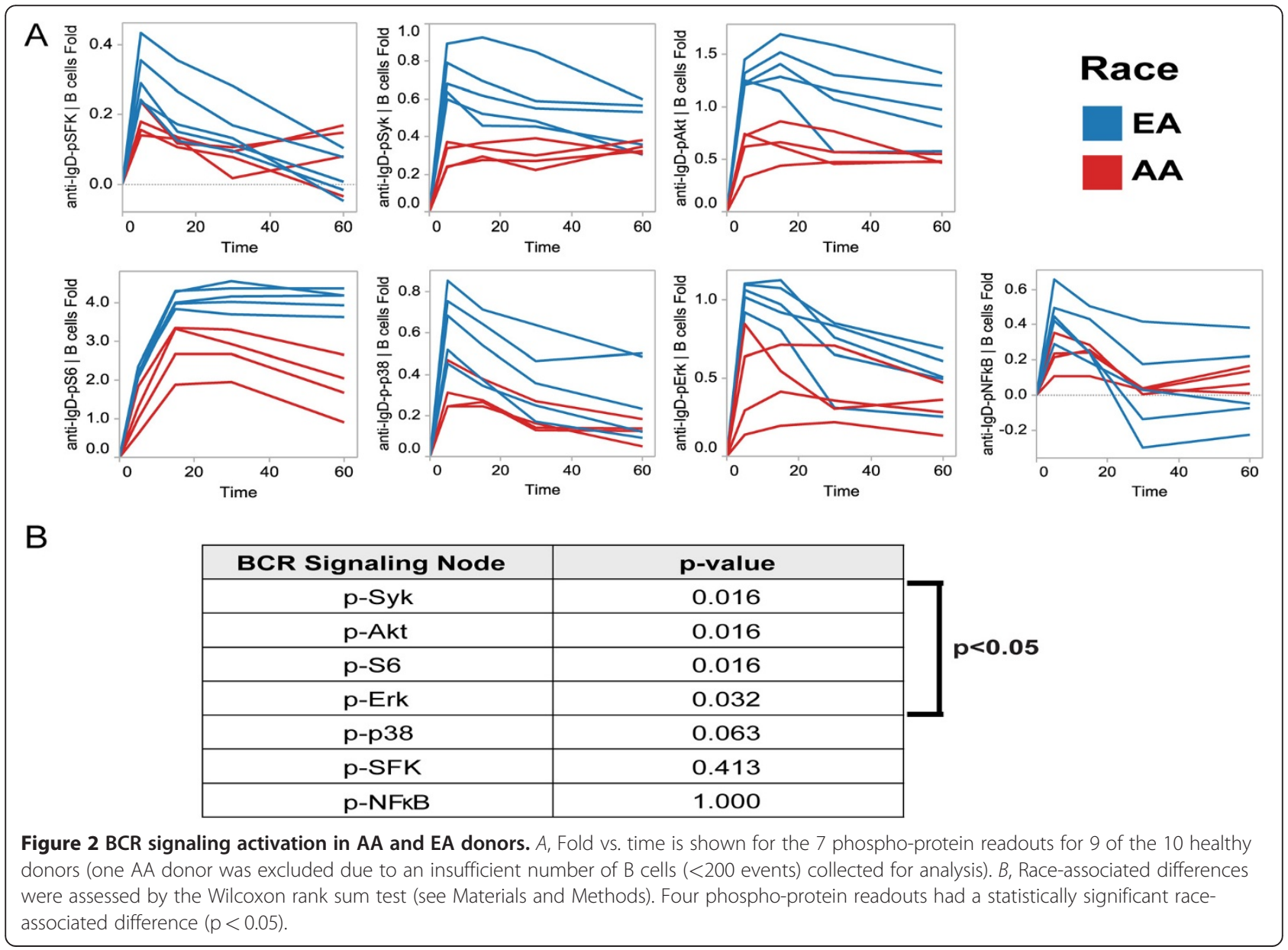




\section{Race-associated differences in BCR signaling pathway activation}

On average, compared with B cells from EA donors, B cells from AA donors displayed lower anti-IgDinduced phosphorylation for all 7 of the tested BCR pathway components (Figure 2A). For the p-Syk, p$\mathrm{S} 6$, p-Akt, and p-Erk signaling readouts, the raceassociated difference in the magnitude of anti-IgDinduced activation levels (quantified by the Fold and averaged over all time points, see Materials and Methods) reached statistical significance $(\mathrm{p}<0.05$, Wilcoxon test, Figure 2B). To assess whether or not the race-associated difference in BCR pathway activation is specific to modulation with anti-IgD, the 7 phospho-proteins were measured following modulation with anti-IgM (5') and higher induced phosphorylation was observed in EA donors than in AA donors, reaching statistical significance $(p<0.05$, Wilcoxon test) for p-Syk, p-S6, p-Akt, and p-SFK (data not shown). Future studies will be performed in larger independent donor cohorts with greater statistical power to assess the external validity of the race-associated differences observed in this exploratory study.

\section{BCR signaling kinetics in AA and EA donors}

For the BCR signaling pathway components p-SFK, p$\mathrm{p} 38$, and $\mathrm{p}-\mathrm{NF}-\mathrm{kB}$, a rapid, transient increase in signal was observed, peaking at the 5 minute time point followed by a steady decrease until the 60 minute time point. For these three BCR signaling components, the kinetic profiles were comparable between AA and EA donors. Previous studies have reported similar kinetic profiles for p-p38 in human CD20+ B cells following BCR cross-linking with anti-IgM and anti-IgG [10].

In contrast, anti-IgD-induced signaling kinetics for $\mathrm{p}$ Syk, p-Akt, and p-S6 appeared different among EA and AA donors (Figure 2A). Specifically, increases for both p-Syk and p-Akt were at or near peak values at 5 minutes and, in EA donors, gradually declined through the 60 minute time point [with an average difference in the Fold between the $5^{\prime}$ and $60^{\prime}$ time point of 0.25 (for pSyk) and 0.32 (for p-Akt)], while peak levels (although lower in magnitude) were generally sustained through the 60 minute time point in AA donors [with an average difference in the Fold between the 5' and 60' time point of -0.04 (for $\mathrm{p}$-Syk) and 0.11 (for $\mathrm{p}$-Akt)]. In recent analyses using $\mathrm{B}$ cells from healthy donors, antiIgM/anti-IgG-induced Syk phosphorylation was reported

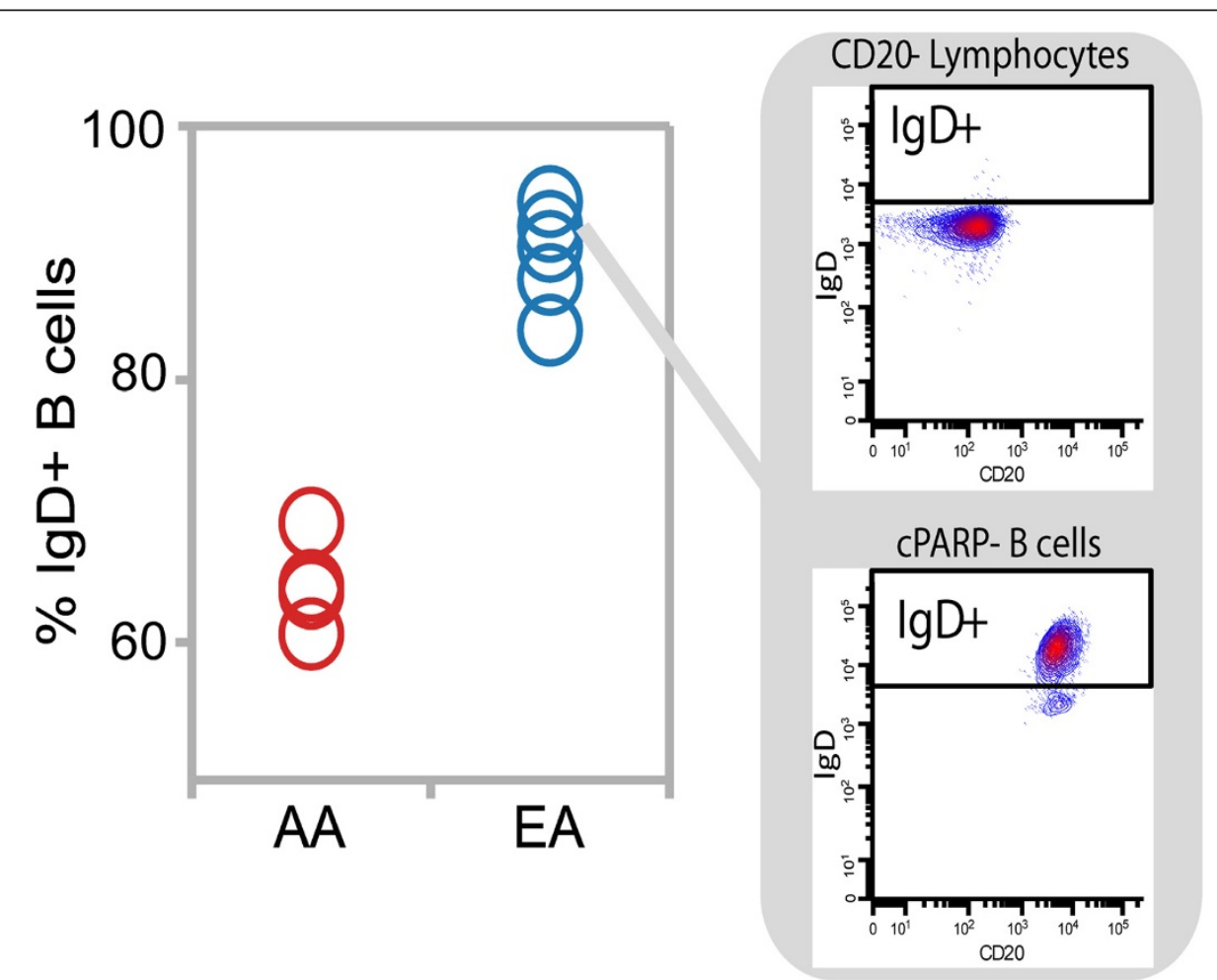

Figure $3 \lg D+B$ cell percentages in AA and EA donors. CD20- lymphocytes were used as an internal negative control to define the lgD+ region for each donor. The IgD+ region was applied to the viable $B$ cell subpopulation to determine the $\lg D+B$ cell percentage for each donor. Representative flow plots from one healthy donor are shown. IgD+B cell percentages were assessed under the unmodulated condition. EAs had a higher percentage of IgD+B cells than AAs and the race-associated difference was significant ( $p=0.016$, Wilcoxon test). 
to peak between 4 and 8 minutes and return to basal levels by 90 minutes [10] thus displaying a signaling kinetic profile similar to the $\mathrm{p}$-Syk profile observed here for EA donors.

For anti-IgD-induced p-S6, peak activity was observed at 15 minutes (i.e. delayed relative to more upstream pathway components p-Akt and p-Syk) and, in EA donors, the p-S6 levels were sustained through the 60 minute time point (with an average difference in the Fold between the 15' and 60' time point of 0.02 ), while $\mathrm{p}$-S6 levels had declined by the 60 minute time point in AA donors (with an average difference in the Fold between the 15' and 60' time point of 0.98 ).

To assess BCR signaling responses at a network level, Pearson correlation coefficients between pairs of signaling nodes at each time point were calculated. All of the responses at the 5' time point were positively correlated $(r>0.6)$, and positive correlations were observed between the majority of the nodes at later time points (i.e. 15, 30', and 60') with the exception of the node-to-node correlations with $\mathrm{p}-\mathrm{NF}-\mathrm{\kappa} \mathrm{B}$ or $\mathrm{p}-\mathrm{SFK}$, nodes which displayed low or no induced activation (average Folds $<0.3$ ) at later time points (Figure 2A).
Race-associated differences in IgD + B cell percentages Given that race-associated differences in anti-IgD responses were observed in the overall viable $B$ cell population, it is of interest to determine if the race-associated difference in anti-IgD responses is due to a raceassociated difference in anti-IgD responses specifically within the IgD $+B$ cell subpopulation and/or due to a race-associated difference in the frequency of $\operatorname{IgD}+\mathrm{B}$ cells. Although it would be ideal to measure anti-IgD responses directly within IgD $+B$ cells, modulation with anti-IgD can interfere with the subsequent detection of surface IgD with fluorescently-labeled anti-IgD. Therefore, an indirect approach was taken whereby the IgD+ B cell frequency was determined in the unmodulated condition and the relationship between $\operatorname{IgD}+\mathrm{B}$ cell frequencies and anti-IgD responses within the overall viable $B$ cell population was assessed.

The percentage of IgD $+B$ cells was determined for each donor by using the CD20- Lymphocyte subpopulation within each donor PBMC sample as an internal negative control to define an IgD+ region which was subsequently applied to the viable B cell subpopulation (Figure 3). The percentage of IgD $+\mathrm{B}$ cells was significantly lower in AA donor samples than in EA donor samples $(p=0.016$, Wilcoxon test). Despite a significant

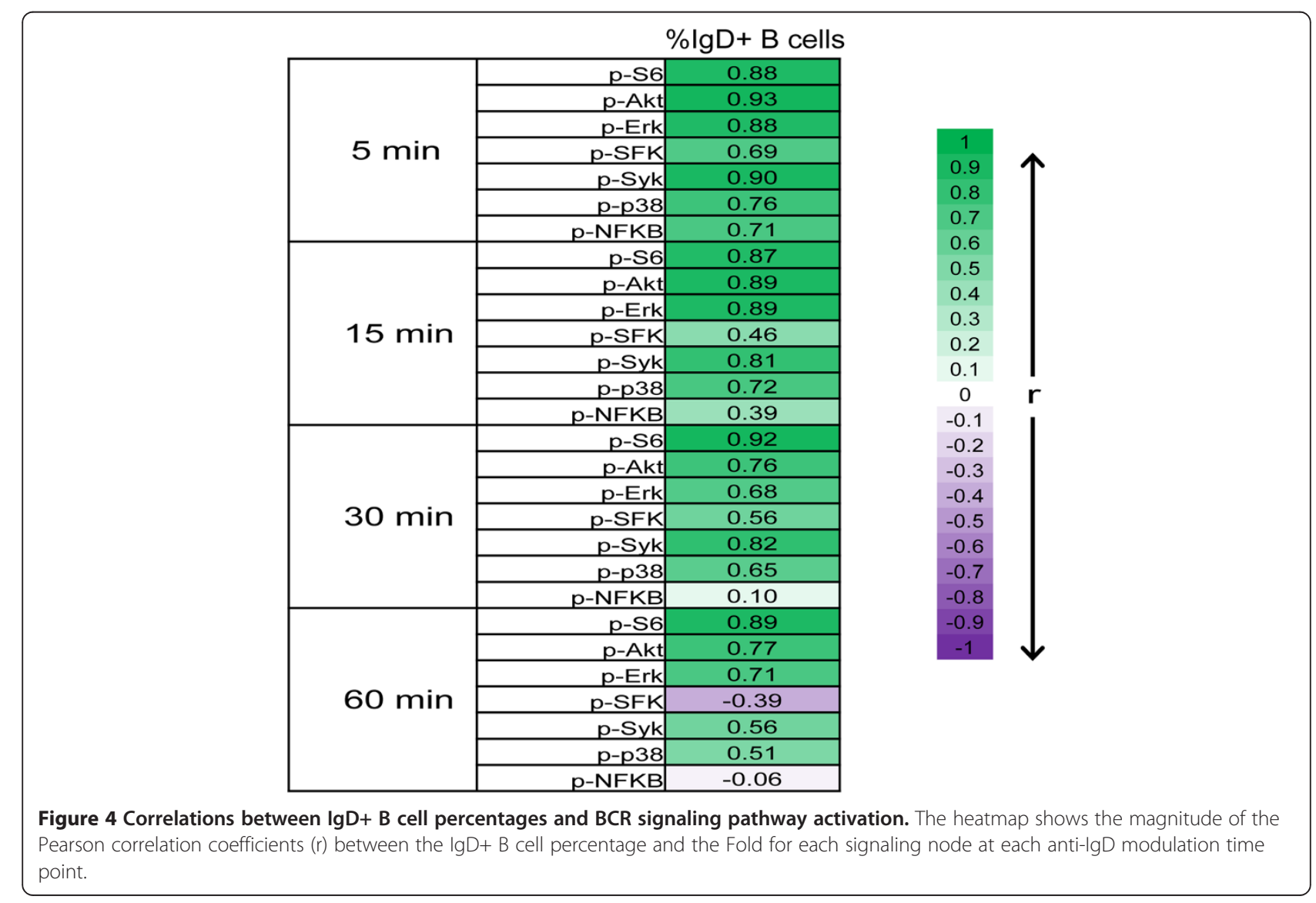


race-associated difference in the percentage of $\operatorname{IgD}+\mathrm{B}$ cells, the level of IgD expression within the IgD+ B cell subset (as quantified by the median fluorescence intensity (MFI) of IgD in the IgD+ B cell subset) did not differ between the races $(p=0.286$, Wilcoxon test).

The correlation between IgD+ B cell frequency and $\mathrm{BCR}$ signaling pathway activation was then assessed by calculating the Pearson correlation coefficient, $r$, between IgD $+B$ cell percentage and the Fold metric for each $\mathrm{BCR}$ signaling node at each anti-IgD modulation time point (Figure 4). A positive correlation (defined as $r$ $>0.6$ ) was observed for the majority of BCR signaling responses measured, with the exception of $\mathrm{p}-\mathrm{NF}-\mathrm{KB}$ and p-SFK at the 15, 30, and 60', [low or no induced activation (average Folds $<0.3$ ) was observed for these nodes at these time points (see Figure 2A)].

For the anti-IgD induced p-S6 response at the 15' time point, pronounced bimodality was observed allowing for a quantitative assessment of the percentage of p-S6 "high" (i.e. anti-IgD responsive $\mathrm{B}$ cells). The percentage of antiIgD responsive $\mathrm{B}$ cells closely approximated the percentage of IgD+ B cells for each donor (Figure 5). While the percentage of p-S6 "high" cells was significantly different between the two racial groups $(p=0.016$, Wilcoxon test), the ratio of the MFI in the p-S6 "high" region to the MFI in the p-S6 "low" region did not differ between the races ( $\mathrm{p}=0.905$, Wilcoxon test).

Overall, these results suggest that the observed raceassociated difference in BCR signaling pathway activation could be attributed at least in part to a race-associated difference in IgD+ B cell frequencies (and thus the percentage of $\mathrm{B}$ cells that respond to anti-IgD) and not to surface levels of IgD or intrinsic differences in the magnitude of the anti-IgD induced response within each B cell.

Within the peripheral blood, the IgD+ B cell subpopulation consists of naïve (CD27-) B cells and non-switched (IgE-/IgG-/IgA-) memory (CD27+) B cells, while the IgD- B cell subpopulation consists primarily of switched $(\operatorname{IgE}+/ \operatorname{IgG}+/ \operatorname{IgA}+)$ memory $(\mathrm{CD} 27+)$ B cells $[11,12]$. Thus, in the donor set studied here, AAs may have a greater frequency of switched memory B cells than EA donors. Studies which include additional B cell phenotypic markers, such as CD27 and IgM, are currently underway to assess race-related differences in more welldefined B cell subsets. To our knowledge, no prior studies have reported race-associated differences in B cell subset frequencies. However, changes in the composition of the peripheral B cell pool have been reported for systemic lupus erythematosus [13], common variable immunodeficiency [14], and with age [15].

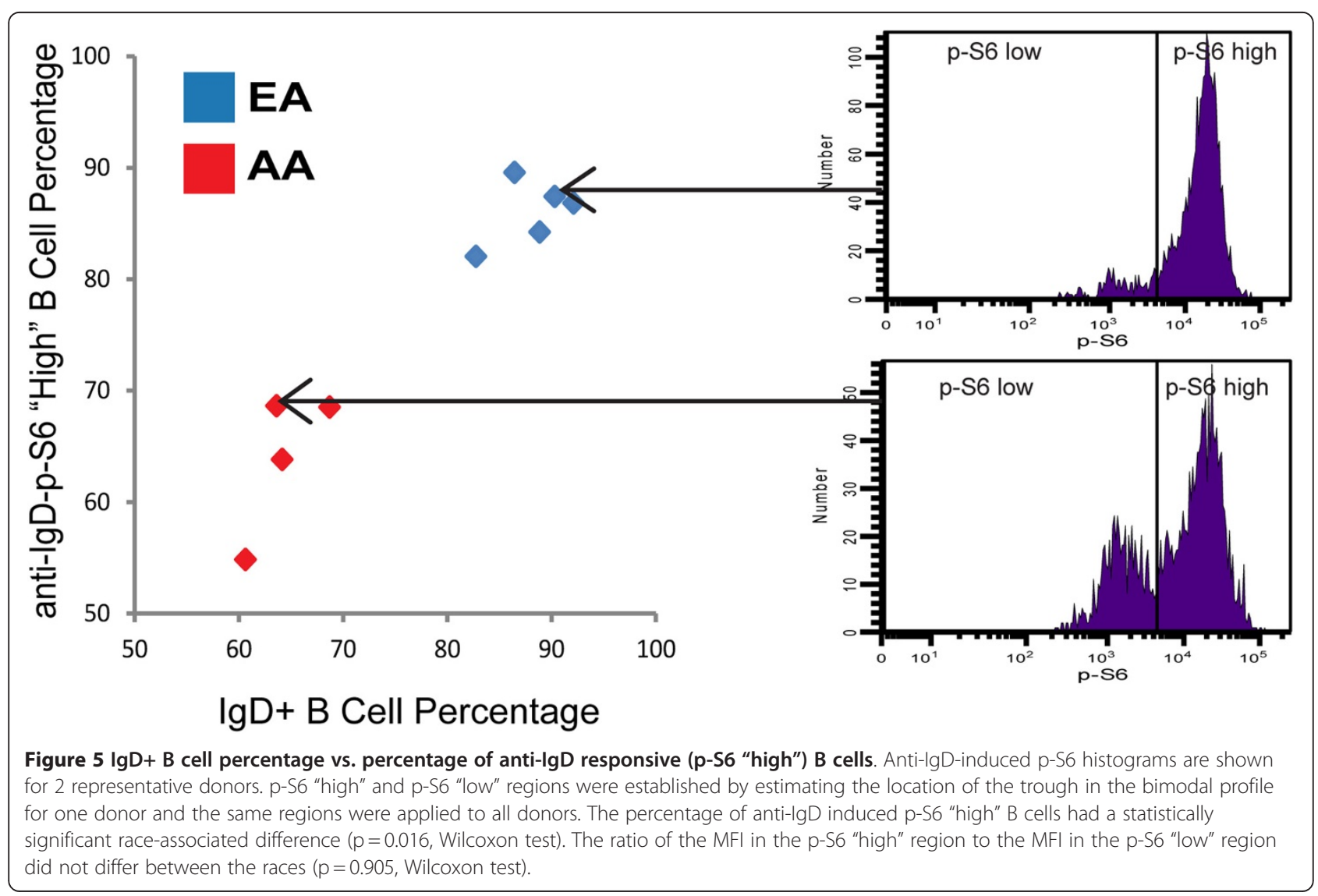


Establishing the range of $\mathrm{BCR}$ signaling responses in healthy donors can enable the identification of dysregulated signaling in disease states by providing a reference range to which signaling responses from diseased samples can be compared. Recently, BCR signaling profiles from follicular lymphoma B cells were compared with signaling profiles from B cells from healthy blood donors to identify alterations in BCR-mediated signaling in follicular lymphoma, and BCR-mediated phosphorylation of p38 was found to be lower in follicular lymphoma than in a reference healthy blood donor [16]. The data presented here demonstrating that BCR-mediated signaling within healthy donors can vary with at least one demographic variable (race) highlights the importance of using healthy donor cohorts consisting of individuals matched to the diseased population by demographic characteristics such as age, race, and gender. Such criteria may be critical in establishing reference signaling ranges as a basis for comparison with diseased signaling responses.

\section{Conclusions}

In this study, differences in B cell subset frequencies and BCR-mediated signaling pathway activation were found amongst AA and EA donors. Subsequent studies will extend the SCNP approach used here to assess differences in B cell subpopulation frequencies and BCR signaling in additional ethnic groups. Controlling for ethnicity is emerging as a key component in assuring the accuracy of clinical diagnostics [17] and in selecting treatments [4]. Ultimately, a greater understanding of race-associated differences in immune cell signaling responses may help to elucidate the basis for racial differences in immunemediated disease prevalence and treatment response rates.

\section{Abbreviations \\ AA: African American; EA: European American; SCNP: Single-cell network profiling.}

\section{Competing interests}

$\mathrm{DML}, \mathrm{BL}, \mathrm{REH}$, and $\mathrm{AC}$ are employees of Nodality Inc. KM is an employee of Medlmmune. The other authors declare no financial conflicts of interest.

\section{Acknowledgments}

We thank all donors who have donated samples for this study.

\section{Author details}

${ }^{1}$ Nodality, South San Francisco, CA 94080, USA. ${ }^{2}$ Medlmmune, Mountain View, CA 94043, USA. ${ }^{3}$ Department of Genetics, Cell and Immunobiology, Semmelweis University, Budapest, $\mathrm{H}-1089$, Hungary. ${ }^{4}$ Infectious Disease and Immunogenetics Section, Department of Transfusion Medicine, Clinical Center, and Center for Human Immunology, National Institutes of Health, Bethesda, MD 20892, USA.

\section{Authors' contributions}

$D M L, Z P, E W, R E H, K M, F M M$, and AC conceived the study. DML and KM performed the experiments. DML and BL analyzed the experiments and drafted the manuscript. All authors read and approved the final manuscript.
Received: 14 April 2012 Accepted: 6 June 2012

Published: 6 June 2012

\section{References}

1. McCarty DJ, Manzi S, Medsger TA Jr, Ramsey-Goldman R, LaPorte RE, Kwoh CK: Incidence of systemic lupus erythematosus. Race and gender differences. Arthritis Rheum 1995, 38:1260-1270.

2. Rosati G: The prevalence of multiple sclerosis in the world: an update. Neurol Sci 2001, 22:117-139.

3. Ge D, Fellay J, Thompson AJ, Simon JS, Shianna KV, Urban TJ, Heinzen EL, et al: Genetic variation in IL28B predicts hepatitis $C$ treatment-induced viral clearance. Nature 2009, 461:399-401.

4. Ratner M: Benlysta makes history. Nat Biotechnol 2011, 29:298.

5. Cesano A, Rosen DB, O'Meara P, Putta S, Gayko U, Spellmeyer DC, Cripe LD, et al: Functional pathway analysis in acute myeloid leukemia using single cell network profiling assay: Effect of specimen source (bone marrow or peripheral blood) on assay readouts. Cytometry B Clin Cytom 2012, doi:10.1002/cyto.b.21007.

6. Longo DM, Louie B, Putta S, Evensen E, Ptacek J, Cordeiro J, Wang E, et al: Single-cell network profiling of peripheral blood mononuclear cells from healthy donors reveals age- and race-associated differences in immune signaling pathway activation. J Immunol 2012, 188:1717-1725.

7. Longo DM, Louie B, Mathi K, Pos Z, Wang E, Hawtin RE, Marincola FM, Cesano A: Single-cell network profiling (SCNP) reveals race-associated differences in $\mathrm{B}$ cell receptor signaling pathway activation. Blood (ASH Annual Meeting Abstracts) 2011, 118:1125.

8. Pos Z, Selleri S, Spivey TL, Wang JK, Liu H, Worschech A, Sabatino M, et al: Genomic scale analysis of racial impact on response to IFN-alpha. Proc Natl Acad Sci USA 2010, 107:803-808.

9. Krutzik PO, Nolan GP: Intracellular phospho-protein staining techniques for flow cytometry: monitoring single cell signaling events. Cytometry $A$ 2003, 55:61-70.

10. Irish JM, Czerwinski DK, Nolan GP, Levy R: Kinetics of B cell receptor signaling in human $B$ cell subsets mapped by phosphospecific flow cytometry. J Immunol 2006, 177:1581-1589.

11. Klein U, Rajewsky K, Küppers R: Human immunoglobulin (lg) $M+\lg D+$ peripheral blood $B$ cells expressing the CD27 cell surface antigen carry somatically mutated variable region genes: CD27 as a general marker for somatically mutated (memory) B cells. J Exp Med 1998, 188:1679-1689.

12. Warnatz K, Schlesier M: Flowcytometric phenotyping of common variable immunodeficiency. Cytometry B Clin Cytom 2008, 74:261-271.

13. Rodríguez-Bayona B, Ramos-Amaya A, Pérez-Venegas JJ, Rodríguez C, Brieva $J A$ : Decreased frequency and activated phenotype of blood CD27 IgD IgM B lymphocytes is a permanent abnormality in systemic lupus erythematosus patients. Arthritis Res Ther 2010, 12:R108.

14. Warnatz K, Denz A, Dräger R, Braun M, Groth C, Wolff-Vorbeck G, Eibel H, et al: Severe deficiency of switched memory B cells $(C D 27(+) \operatorname{lgM}(-) \lg D(-))$ in subgroups of patients with common variable immunodeficiency: a new approach to classify a heterogeneous disease. Blood 2002, 99:15441551.

15. Chong $Y$, Ikematsu H, Yamaji K, Nishimura M, Nabeshima S, Kashiwagi S, Hayashi J: CD27(+) (memory) B cell decrease and apoptosis-resistant CD27(-) (naive) B cell increase in aged humans: implications for agerelated peripheral B cell developmental disturbances. Int Immunol 2005, 17:383-390.

16. Irish JM, Czerwinski DK, Nolan GP, Levy R: Altered B-cell receptor signaling kinetics distinguish human follicular lymphoma B cells from tumorinfiltrating nonmalignant B cells. Blood 2006, 108:3135-3142.

17. Moore B, Hu H, Singleton M, Reese MG, De La Vega FM, Yandell M: Global analysis of disease-related DNA sequence variation in 10 healthy individuals: implications for whole genome-based clinical diagnostics. Genet Med 2011, 13:210-217.

doi:10.1186/1479-5876-10-113

Cite this article as: Longo et al:: Racial differences in B cell receptor signaling pathway activation. Journal of Translational Medicine 2012 10:113. 